\title{
A MODERATELY HIGH PROTEIN DIET IS AS EFFECTIVE DURING NEOADJUVANT CHEMOTHERAPY AS IT IS 2 YEARS AFTER BREAST CANCER SURGERY - A PILOT TRIAL
}

Diana Viorela Artene', ND, MSN, Cristian Ioan Bordea', MD, PhD,
Prof. Dr. Alexandru Blidaru', MD, PhD
'Oncology Nutrition, "Carol Davila" Medicine University, Bucharest, Romania
${ }^{2}$ Department II of Surgical Oncology, "Alexandru Trestioreanu” Institute of Oncology, Bucharest, Romania
${ }^{3}$ Department of Surgery, "Carol Davila" Medicine University, Head of Surgical Oncology Department at
"Alexandru Trestioreanu” Institute of Oncology, Bucharest, Romania

\begin{abstract}
Introduction. Statistically, patients who achieve and maintain their optimal weight during breast cancer treatment have the best prognostic. Breast cancer associated weight gain starts during neoadjuvant chemotherapy when many patients either eat too little because of the decreased appetite or eat too much to emotionally cope with the treatment.

Materials and method. To test if we can prevent weight gain during neoadjuvant chemotherapy we assigned 46 patients during neoadjuvant chemotherapy $(\mathrm{CH})$ and of 50 patients 2 years after surgery (S) to follow a moderately high protein diet - based on eating only when hungry foods naturally high in protein, high quality fatty acids and carbohydrates, calcium, pre- and probiotics. We used no dietary supplements.

We measured weight (W), subcutaneous fat (\%SF) and visceral fat percentages (\% VF) with a multi-frequency BIA scale at the beginning of the study and after 12 weeks of intervention (time needed to administrate 4 chemotherapy sessions). To validate BIA measurements, we also measured waist and hips circumferences. Results. Although S patients obtained better results on all measured parameters, $\mathrm{CH}$ patients also improved their body composition despite chemotherapy administration during the trial: $1.611 \pm 2.89 \% \mathrm{~kg}(p=0.005)$, $1.79 \pm 3.32 \%$ subcutaneous fat $(p=0.006), 0.4 \pm 0,81 \%$ visceral fat $(p=0.012)$ and had a $0.29 \pm 0.4$ in the waist to hips ratio $(p=0.001)$.

Conclusion. A moderately lower carb diet is as effective for preventing sarcopenic obesity during chemotherapy as it is 2 years after surgery thus sarcopenic obesity prevention can begin from as early as diagnosis.
\end{abstract}

Keywords: oncology nutrition, breast cancer, sarcopenic obesity neoadjuvant chemotherapy

\section{INTRODUCTION}

Many $\mathrm{ER}+/ \mathrm{PR} \pm / \mathrm{HER} 2$ - breast cancer patients gain weight during chemotherapy potentially decreasing treatment efficacy and increasing recurrence and oncology specific mortality risks. (1)

- Neoadjuvant chemotherapy is less effective in overweight and obese patients, even with early-stage breast cancers $(2,3)$;

- Weight gain during chemotherapy increases the risk of recurrence and mortality, the best prognosis being obtained by patients who manage to maintain their body weight throughout chemotherapy (4);

- Obesity increases the risk of pulmonary and liver metastasis (5-8);
- Obesity decreases younger breast cancer patients' survival (9).

Studies prove that sarcopenic obesity developed during breast cancer chemotherapy is still present 3 years after chemotherapy if no nutritional intervention is prescribed to the patients (10-12).

There are 2 main mechanisms behind this weight: decreased metabolism generated by a too low protein intake or by treatment induced sarcopenia and decreased ability to perceive satiety generated by stress disturbed eating behaviour.

In theory, weight loss is simple: we decrease food intake thus we lose weight. And it is true: the total body weight decreases by caloric restriction but only initially and only if the caloric restriction 
falls below basal metabolism. The weight loss obtain through caloric restriction can be maintain only with a higher caloric restriction: the less you eat to lose weight, the less you can eat without gaining weight.

Obesity solution is more complicated than "eating less" because fat gain etiology involves:

- insulin resistance generated either by overeating (13), either by starvation lipolysis (14);

- then insulin stimulates the already increased adipocytes' leptin secretion leading to hypothalamic leptin resistance (15);

- leptin resistance leads to hedonic eating (16);

- then hedonic eating decreases sleep quality because when we do not respect satiety and continue eating after feeling it, its main neuromediator - POMC is transformed not only into the beta-endorphins - responsible for the emotional numbness fell after overeating, but also into $\mathrm{CRH}$ and into the alfa-MSH hormone - both involved into the fight, flight or freeze stress responses (17);

- And then after a bad sleep, the patient moves less and eats more fattening foods - behavior generating insulin resistance etiology. And so, the cycle restarts all over again leaving no room for the eating less theory (18).

Eating less than the basal metabolic needs deregulates the main appetite hormones: ghrelin and leptin putting the patient in a worse body composition than before the diet - simply because it generates dehydration and muscle loss, not fat loss.

Initially caloric restriction increases ghrelin and decreases leptin because of the glucagon commanded lipolysis. But glucagon also stimulates insulin secretion. Insulin inhibits lipolysis and stimulates back the leptin secretion to maintain fat stores during insufficient energy intake.

Then the miocytes membrane are kept close for any available glucose because they are not essential for survival and the patients start losing muscle proteins for gluconeogenesis. Because of the decreased muscle insulin sensitivity, nutrients that should have fed the skeletal muscle are stored into the increasing fat tissue. So gradually - what the restricted food intake that generated weight loss yesterday becomes more than enough to generate fat gain today.

Moreover, because amygdala, nucleus accumbens and hippocampus regulates eating behavior eating too little builds binge eating behavior by increasing cravings for foods high in fats and carbohydrates. Because of the eating neurophysiology, the other side of caloric restriction is overeating, hypothalamic leptin resistance and then more overeating.

Through metabolic decrease, sarcopenia increases body fat while the patients are still normal weight, then the increased fat secretes more leptin which generates increased cravings and decreased ability to perceive satiety - leading to overeating and fat gain. Then the fat gain gradually becomes visible as the hepatic and muscular insulin sensitivity decreases - and we start recognizing obesity.

Current literature proves that both sarcopenia and overeating can be decreased by a high protein diet, through better muscle protein synthesis/degradation ratio improved satiety.

Also, there are scientists who consider the 15$20 \%$ general recommended protein intake inappropriate for people at risk for sarcopenia $(19,20)$. In 2001 Campbell and colleagues proved that respecting this recommendation lead to sarcopenia. Then in 2007 he proved that a protein intake of $30-35 \%$ successfully counteracted sarcopenia (21).

So, because of malignant metabolism and because of sarcopenia risk, clinical nutrition for breast cancer patients during oncology treatment is based on a moderately higher protein diet. And because insulin resistance doesn't just happen through overeating but also through the hyperlipidemia induced by starvation - the only "less" eating we recommended to our patients is not eating when not hungry (22).

\section{Study Design}

There was no control group because we tested the difference in efficacy of the same diet in patients during two different stages of the $\mathrm{ER}+/ \mathrm{PR} \pm$ / HER2- breast cancer treatment.

We included $46 \mathrm{ER}+/ \mathrm{PR} \pm / \mathrm{HER} 2$ - breast cancer patients during neoadjuvant chemotherapy (,CH” group) and $50 \mathrm{ER}+/ \mathrm{PR} \pm / \mathrm{HER} 2-$ breast cancer patients at 2 years after the surgical treatment (,S" group).

We excluded patients with recurrence and metastasis and those suffering from diabetes, hypothyroidism, kidney disease, osteoporosis, and depression.

We measured weight (W), subcutaneous fat $(\% \mathrm{SF})$ and visceral fat percentages $(\% \mathrm{VF})$ with a multi-frequency BIA scale at the beginning of the study and after 12 weeks of intervention (time needed to administrate 4 chemotherapy sessions). To validate BIA measurements, we also measured waist and hips circumferences.

To monitor patients' compliance with the dietary recommendations, we instructed them to keep 
a daily food $\log$, where they wrote at what time they had each meal, exactly what it contained and in what quantity and if they were hungry or not when they ate.

\section{Intervention}

We gave patients a table where foods were classified as proteins and fats, carbohydrates or fibres supplying sources and they were also taught to eat balanced meals based on proteins and fats, carbohydrates and fibres supplying foods at each meal.

To prevent sarcopenia, to counteract the Warburg, reverse Warburg and Crabtree effects, and to prevent ketosis and de novo lipogenesis we decreased the recommended percentage of carbohydrate intake from the common value recommended for general population - of 55-60\% - to only $40 \%$. We calculated portion size to reach a protein intake of $1.5 \mathrm{~g} / \mathrm{kg}$ body, which meant for most patients a 25-30 g protein intake per meal. Per immunohistochemistry, we recommended a normal fat intake of 25-30\%.

To prevent anaemia, we instructed them to eat foods high in proteins and calcium (yoghurt, sour milk and kephir, raw seeds and nuts) at different meals than foods high in iron (fish, chicken, eggs, beans, chickpeas and other lentils).

To prevent gastrointestinal infections potentially associated with chemotherapy induced dysbiosis we instructed them, to wash very well raw fruits and vegetables, to avoid eating foods containing raw animal ingredients (like unpasteurized ice cream or mayonnaise, sauces, deli meats or cheese, smoked raw fish, canned fish or roe), and to eat at least two fermented dairies portions per day.

To sustain complete fatty acids catabolism for energy, when not hungry, patients could only drink plain water, no snacks, and no drinks other than water. Only one coffee was allowed at the first meal of the day, and tea with other meals, but not in between meals, due to caffeine and theine impact on insulin secretion, and no soft drinks due to their impact on presynaptic dopamine re-transporters and on hypothalamic leptin sensitivity. Also, to ensure a proper gastric emptying time, we recommended minimum $2 \mathrm{~h}$ between taking any meal and sleeping.

To ensure an optimum therapeutic effect during chemotherapy, we asked patients to refrain from eating omega-3 food sources 3 days before, during and 3 days after the day of the chemotherapy administration, to be more physically active, to drink more water and prune juice (the later only at meals) in case of constipation, to eat slowly and to avoid cruciferous vegetables, beans, and milk when feeling bloated, as well as raw fruits, vegetables and coffee in case of diarrhea.

We also asked them to use lemon juice and to try to chill down in case of faintness and dizziness, to avoid hot or very cold foods and drinks, citric fruits and pickles in case of mucositis, and to sip mint lemonade sweetened with brown sugar, spices and aromatic herbs for extra flavour in case of abnormal, metallic taste.

Finally, to avoid phytoestrogen interaction with antiestrogenic treatments, we recommended the avoidance of plant supplements and soy intake.

\section{RESULTS}

We used IBM SPSS software v. 22, two-tailed paired sample $\mathrm{T}$ test to analyse the differences in mean values obtained at the two weight, body composition, and waist circumferences measurements performed for each patient. Statistical significance was considered when $\mathrm{p}$-value was less than 0.01 $(\mathrm{P}<0.01)$.

$15 \mathrm{CH}$ patients and $6 \mathrm{~S}$ patients left the trial because they reported feeling ill.

Remaining patients, both during neoadjuvant chemotherapy and during antiestrogenic treatment obtained statistically significant weight loss (Fig. 1).

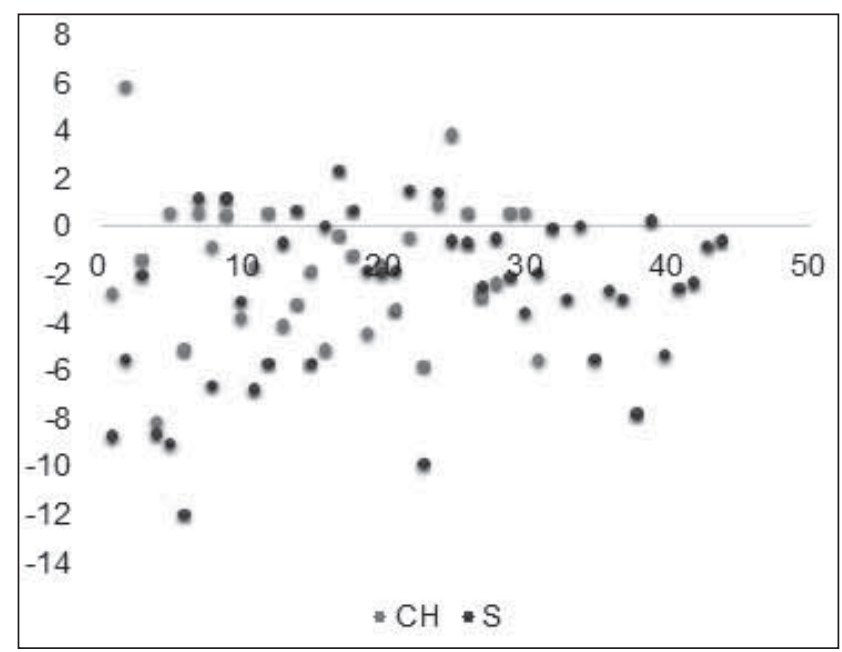

FIGURE 1. Comparative weight evolution for patients during neoadjuvant chemotherapy $(\mathrm{CH})$ and patients during antiestrogenic treatment (S)

Many $\mathrm{CH}$ patients reported that they became more sedentary during treatment administration, and despite feeling ill only for a few days after chemotherapy session administration, only some felt tired for the entire treatment duration. Also, some of neoadjuvant $\mathrm{CH}$ patients also reported sleeping disorders. 
TABLE 1. Comparative weight evolution patients during neoadjuvant chemotherapy and patients during antiestrogenic treatment

\begin{tabular}{|l|c|c|c|c|c|c|c|c|c|}
\hline \multirow{2}{*}{ Group } & \multicolumn{3}{|c|}{ Waist/Hips } & \multicolumn{3}{c|}{ SF\% } & \multicolumn{3}{c|}{ VF\% } \\
\cline { 2 - 10 } & Mean & SD & P & Mean & SD & P & Mean & SD & P \\
\hline CH & 0.29 & 0.40 & 0.001 & 1.79 & 3.32 & 0.006 & 0.40 & 0.81 & 0.012 \\
\hline S & 0.12 & 0.28 & 0.004 & 2.20 & 3.99 & 0.001 & 0.68 & 1.11 & 0.001 \\
\hline
\end{tabular}

On the other hand, most $\mathrm{S}$ patients restarted work and some even practice regular sports activities. The main complain were induced menopause side effects in premenopausal women at the time of the diagnosis and muscle and joint pains in patients under aromatase inhibitors antiestrogenic treatment.

Also, in our patients' waist and hip circumference evolution during the trial matches the fat loss measured with the multi-frequency BIA (Table 1).

\section{DISCUSSIONS}

Many studies prove high protein diet effective to counteract sarcopenia.

For instance, Paddon-Jones et al. proved in 2004 that high protein diet prevent sarcopenia even during a mandatory 28-day bed arrest (20). And in 2007 Campbell and Leidy proved that a high protein diet can prevent sarcopenia during hypocaloric weight loss diets in overweight menopausal women (21). But in all these studies the higher protein intake needed to counteract sarcopenia was covered by dietary protein supplements not by food.

Our study is one of the few nutrition intervention studies that only used foods to counteract chemotherapy associated sarcopenia, and we chose so because of the many metabolic changes that can occur during chemotherapy.

The 2002 analysis published in Journal of Clinical Oncology by Chlebowski, Aiello and McTiernan cites 158 studies that both proves the importance of counteracting sarcopenic obesity during breast cancer treatment and the relative lack of clinical solutions that can be effective in the real-life situations of breast cancer patients (23).

From these cited studies, the one of Camoriano et al done on 545 breast cancer patients proved that an after-diagnosis weight gain of $5.9 \mathrm{~kg}$ increases the risk of recurrence, metastasis and mortality (24). Thus, we consider the minimal $1.6 \mathrm{~kg}$ lost by our overweight and obese patients during chemotherapy as a positive result, potentially improving patients' response to treatment and long term prognosis.

One of the few interventional studies meant to counteract sarcopenic obesity during breast cancer chemotherapy was done by Loprinzi et al at Mayo Clinic (25). The study involved 107 breast cancer patients, half randomized to classic oncology treatment, half to classic oncology treatment $=$ control group + hypocaloric diet $=$ intervention group. After six months, the intervention group gained $2 \mathrm{~kg}$ and the control group gained $3.5 \mathrm{~kg}$.

According to Chlebowski, Aiello and McTiernanthe main limitations of the oncology nutrition studies meant to counteract sarcopenic obesity during breast cancer treatment was the short duration, the low number of participants, the decrease compliance to dietary recommendations and the fact that most proposed solutions would work only in clinical settings and not in outpatient settings.

Our study was a short duration pilot study with a low number of patients but because the proposed solution worked in the real-life conditions, we consider that in the case of breast cancer we can prevent sarcopenic obesity from as early as neoadjuvant chemotherapy using nothing but foods and the patients eating behaviour.

\section{CONCLUSION}

A moderately lower carb diet is as effective for preventing sarcopenic obesity during chemotherapy as it is 2 years after surgery thus sarcopenic obesity prevention can begin from as early as diagnosis.

\section{REFERENCES}

1. Chlebowski R.T., Aiello E., McTiernan A. Weight loss in breast cancer patient management. J Clin Oncol 2002; 20(4): 1128-1143.

2. Litton, Jennifer K., et al. Relationship between obesity and pathologic response to neoadjuvant chemotherapy among women with operable breast cancer. Journal of Clinical Oncology 26.25 (2008): 4072-4077.

3. Ewertz Marianne, et al. Effect of obesity on prognosis after early-stage breast cancer. Journal of Clinical Oncology 29.1 (2010): 25-31.

4. Thivat E., Thérondel S., Lapirot O., Abrial C., Gimbergues P., Gadéa E., Durando X. Weight change during chemotherapy changes 
the prognosis in non metastatic breast cancer for the worse. BMC cancer, 10(1), 648, 2010).

5. Osman Mohammed A., Bryan T. Hennessy. Obesity Correlation With Metastases Development and Response to First-Line Metastatic Chemotherapy in Breast Cancer. Clinical Medicine Insights. Oncology 9 (2014): 105-112.

6. Strong, Amy L., et al. Leptin produced by obese adipose stromal/ stem cells enhances proliferation and metastasis of estrogen receptor positive breast cancers. Breast Cancer Research 17.1 (2015): 112.

7. Dowling R.J.O., et al. Abstract P2-02-09: Obesity associated factors are inversely associated with circulating tumor cells in metastatic breast cancer. (2016): P2-02.

8. Nagahashi M., et al. Abstract P2-05-11: Sphingosine-1-phosphate signaling promotes metastatic niches and lung metastasis in obesity-related breast cancer. (2016): P2-05.

9. Copson E.R., et al. Obesity and the outcome of young breast cancer patients in the UK: the POSH study. Annals of Oncology 26.1 (2015): 101-112.

10. Demark-Wahnefried W., Peterson B.L., Winer E.P., Marks L., Aziz N., Marcom P.K., Rimer B.K. Changes in weight, body composition, and factors influencing energy balance among premenopausal breast cancer patients receiving adjuvant chemotherapy. Journal of clinical oncology, 19(9), 2381-2389, 2001.

11. Ganz P.A., Kwan L., Stanton A.L., et al. Physical and psychosocial recovery in the year after primary treatment of breast cancer. $J$ Clin Oncol 29(9):1101-1109, 2011.

12. Heideman W.H., Russell N.S., Gundy C., et al. The frequency, magnitude and timing of post-diagnosis body weight gain in Dutch breast cancer survivors. Eur J Cancer 45(1):119-126, 2009.

13. Danielsson Anna, et al. Short-term overeating induces insulin resistance in fat cells in lean human subjects. Molecular medicine (Cambridge, Mass. Print) 15.7-8 (2009): 228-234.

14. Koves Timothy R., et al. Mitochondrial overload and incomplete fatty acid oxidation contribute to skeletal muscle insulin resistance. Cell metabolism 7.1 (2008): 45-56.

15. Wang Jiali, et al. Overfeeding rapidly induces leptin and insulin resistance. Diabetes 50.12 (2001): 2786-2791.
16. Zheng Huiyuan, et al. Appetite control and energy balance regulation in the modern world: reward-driven brain overrides repletion signals. International journal of obesity 33 (2009): S8-S13.

17. Lutter M., Nestler E.J. Homeostatic and hedonic signals interact in the regulation of food intake. The Journal of nutrition, 139(3), 629-632, (2009).

18. Markwald, Rachel R., et al. Impact of insufficient sleep on total daily energy expenditure, food intake, and weight gain. Proceedings of the National Academy of Sciences 110.14 (2013): 5695-5700.

19. Campbel W.W., Trappe T.A., Wolfe R.R., Evans W.J. The recommended dietary allowance for protein may not be adequate for older people to maintain skeletal muscle. The Journals of Gerontology Series A: Biological Sciences and Medical Sciences, 56(6), M373-M380, 2001.

20. Paddon-Jones D., Sheffield-Moore M., Zhang X.J., Volpi E., Wolf S.E., Aarsland A., Ferrando A.A., Wolfe R.R. Amino acid ingestion improves muscle protein synthesis in the young and elderly. Am J Physiol Endocrinol Metab. 2004; 286: E321-328.

21. Leidy H.J., Carnell N.S., Mattes R.D., Campbell W.W. Higher protein intake preserves lean mass and satiety with weight loss in pre-obese and obese women. Obesity (Silver Spring) 2007; 15:421-429.

22. Ciampolini M., et al. Hunger can be taught: hunger recognition regulates eating and improves energy balance. International journal of general medicine, 6 (2013): 465.

23. Chlebowski Rowan T., Erin Aiello, Anne McTiernan. Weight loss in breast cancer patient management. Journal of clinical oncology 20.4 (2002): 1128-1143.

24. Camoriano J.K., Loprinzi C.L., Ingle J.N., Therneau T.M., Krook J.E., Veeder M.H. Weight change in women treated with adjuvant therapy or observed following mastectomy for node-positive breast cancer. Journal of Clinical Oncology, 8(8), 1327-1334, 1990.

25. Loprinzi, Charles Lawrence, et al. Randomized trial of dietician counseling to try to prevent weight gain associated with breast cancer adjuvant chemotherapy. Oncology 53.3 (1996): 228-232. 